\title{
Kaon semileptonic form factors in QCD with exact chiral symmetry
}

\section{JLQCD Collaboration: T. Kaneko ${ }^{* a, b} ;$ S. Aoki ${ }^{c}$, G. Cossu ${ }^{a}$, X. Feng ${ }^{a}$, H. Fukaya ${ }^{d}$, S. Hashimoto ${ }^{a, b}$, J. Noaki $^{a}$ and T. Onogi ${ }^{d}$}

${ }^{a}$ High Energy Accelerator Research Organization (KEK), Ibaraki 305-0801, Japan

${ }^{b}$ School of High Energy Accelerator Science, The Graduate University for Advanced Studies (Sokendai), Ibaraki 305-0801, Japan

${ }^{c}$ Graduate School of Pure and Applied Sciences, University of Tsukuba, Ibaraki 305-8571, Japan

${ }^{d}$ Department of Physics, Osaka University, Toyonaka, Osaka 560-0043 Japan

\begin{abstract}
We report on our calculation of the kaon semileptonic form factors in $N_{f}=2+1$ lattice QCD. Chiral symmetry is exactly preserved by using the overlap quark action for a straightforward comparison with chiral perturbation theory (ChPT). We simulate three pion masses down to $290 \mathrm{MeV}$ at a single lattice spacing of $0.11 \mathrm{fm}$ and at a strange quark mass very close to its physical value. The form factors near zero momentum transfer are precisely calculated by using the all-to-all propagator and twisted boundary conditions. We compare the normalizations and slopes of the form factors with ChPT and experiments.
\end{abstract}

The XXIX International Symposium on Lattice Field Theory - Lattice 2011

July 10-16, 2011

Squaw Valley, Lake Tahoe, California

\footnotetext{
*Speaker.

†E-mail: takashi.kaneko@kek.jp
} 


\section{Numerical simulations}

The matrix element of the $K \rightarrow \pi l v$ decays is parametrized by two form factors

$$
\left\langle\pi\left(p^{\prime}\right)\left|V_{\mu}\right| K(p)\right\rangle=\left(p+p^{\prime}\right)_{\mu} f_{+}\left(q^{2}\right)+\left(p-p^{\prime}\right)_{\mu} f_{-}\left(q^{2}\right) \quad\left(q^{2}=\left(p-p^{\prime}\right)^{2}\right) .
$$

The normalization of the vector form factor $f_{+}(0)$ is an important quantity for a precise determination of a CKM matrix element $\left|V_{u s}\right|$ and the search for new physics. For a reliable lattice calculation of $f_{+}(0)$, we also examine the consistency of other information in the matrix element, namely $f_{-}(0)$ and the form factors' shape, with chiral perturbation theory (ChPT) and experiments.

In this article, we report on our calculation of $f_{\{+,-\}}\left(q^{2}\right)$ in $N_{f}=2+1$ QCD. Chiral symmetry is exactly preserved by using the overlap quark action for a straightforward comparison with ChPT. At a single lattice spacing $a=0.112(1) \mathrm{fm}$, we simulate three values of degenerate up and down quark masses $m_{u d}=0.015,0.035$ and 0.050 that cover a range of the pion mass $290-540 \mathrm{MeV}$. The strange quark mass is fixed to a single value $m_{s}=0.080$, which is very close to its physical value $m_{s, \text { phys }}=0.081$. We choose a lattice size, $(L / a)^{3} \times(T / a)=16^{3} \times 48$ or $24^{3} \times 48$, depending on $m_{u d}$ in order to satisfy a condition $M_{\pi} L \gtrsim 4$ to control finite volume effects. The statistics are 2,500 HMC trajectories at each combination of $m_{u d}$ and $m_{s}$.

We calculate two- and three-point functions

$$
\begin{gathered}
C^{P}(\Delta t, \mathbf{p})=\frac{a^{4}}{L^{3} T} \sum_{\mathbf{x}, t} \sum_{\mathbf{x}^{\prime}}\left\langle\mathscr{O}_{P}\left(\mathbf{x}^{\prime}, t+\Delta t\right) \mathscr{O}_{P}^{\dagger}(\mathbf{x}, t)\right\rangle, \\
C_{\mu}^{P Q}\left(\Delta t, \Delta t^{\prime} ; \mathbf{p}, \mathbf{p}^{\prime}\right)=\frac{a^{4}}{L^{3} T} \sum_{\mathbf{x}, t} \sum_{\mathbf{x}^{\prime \prime}, \mathbf{x}^{\prime}}\left\langle\mathscr{O}_{Q}\left(\mathbf{x}^{\prime \prime}, t+\Delta t+\Delta t^{\prime}\right) V_{\mu}\left(\mathbf{x}^{\prime}, t+\Delta t\right) \mathscr{O}_{P}^{\dagger}(\mathbf{x}, t)\right\rangle
\end{gathered}
$$

using an exponential smearing function $\phi(\mathbf{r})=e^{-0.4|\mathbf{r}|}$ for the interpolating operator $\mathscr{O}_{P}^{\dagger}(\mathbf{x}, t)=$ $\sum_{\mathbf{r}} \phi(\mathbf{r}) \bar{q}(\mathbf{x}+\mathbf{r}, t) \gamma_{5} q^{\prime}(\mathbf{x}, t)(P=\pi$ or $K)$. We refer readers to Refs. [U, ㅁ] for details on how to construct these correlators using the all-to-all propagator [B]].

In order to explore the most important kinematical region $q^{2} \sim 0$, the meson momentum $\mathbf{p}^{(\prime)}$ is induced by employing the twisted boundary conditions (TBCs) [四]

$$
q(\mathbf{x}+L \hat{k}, t)=e^{i \theta} q(\mathbf{x}, t), \quad \bar{q}(\mathbf{x}+L \hat{k}, t)=e^{-i \theta} \bar{q}(\mathbf{x}, t) \quad(k=1,2,3),
$$

where $\hat{k}$ is a unit vector in the $k$ direction. We use a common twist angle $\theta$ in all the spatial directions for simplicity. Here we consider the $K^{+} \rightarrow \pi^{0} l v$ channel, and impose the TBCs for the up and strange quarks. The periodic boundary condition is used for the spectator down quark. Our simulation parameters are summarized in Table $\mathbb{W}$.

Table 1: Simulation parameters. We denote the bare quark masses in lattice units by $m_{\{u d, s\}}$.

\begin{tabular}{lllll}
\hline$m_{u d}$ & $m_{s}$ & lattice & $M_{\pi} L$ & $\theta$ \\
\hline 0.050 & 0.080 & $16^{3} \times 48$ & 4.9 & $0.00,0.40,0.96,1.60$ \\
0.035 & 0.080 & $16^{3} \times 48$ & 4.1 & $0.00,0.60,1.28,1.76$ \\
0.015 & 0.080 & $24^{3} \times 48$ & 4.2 & $0.00,1.68,2.64$ \\
\hline
\end{tabular}



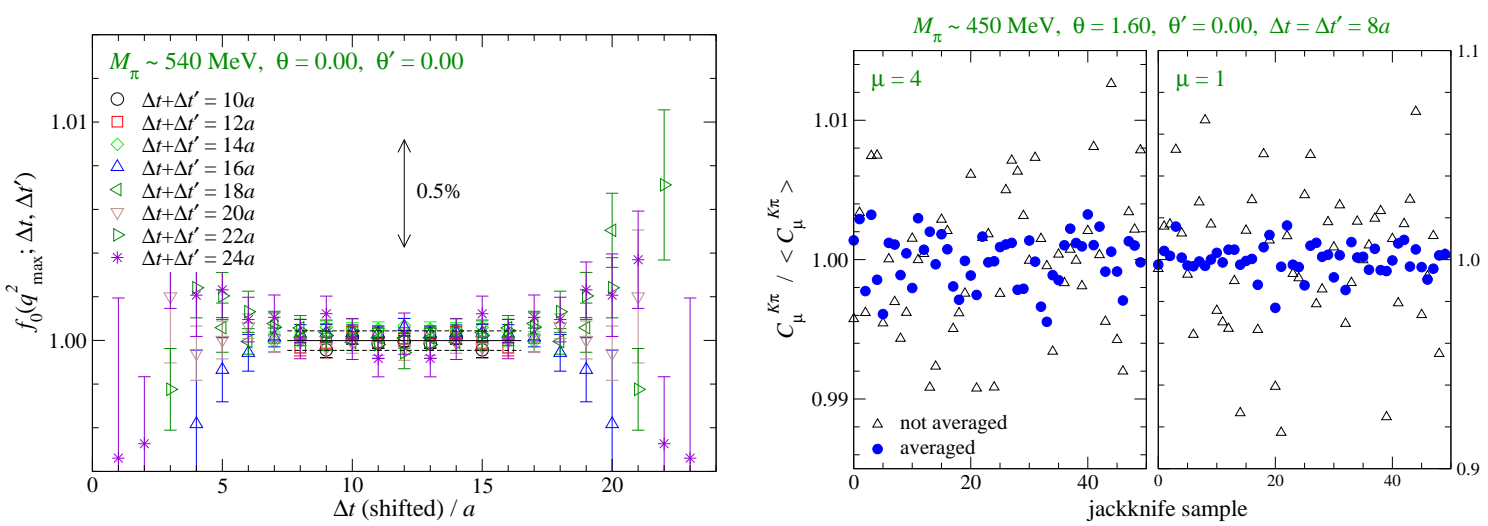

Figure 1: Left panel: effective value $f_{0}\left(q_{\max }^{2} ; \Delta t, \Delta t^{\prime}\right)=2 \sqrt{M_{K} M_{\pi} R\left(\Delta t, \Delta t^{\prime}\right)} /\left(M_{K}+M_{\pi}\right)$ obtained with different values of $\Delta t+\Delta t^{\prime}$. Data are shifted in the horizontal direction so that the meson source and sink operators are located at $T / 4-\left(\Delta t+\Delta t^{\prime}\right) / 2$ and $T / 4+\left(\Delta t+\Delta t^{\prime}\right) / 2$, respectively. Right two panels: threepoint function $C_{4}^{K \pi}$ for each jackknife sample. We plot data normalized by the Monte Carlo average for $\mu=4$ and 1 in each panel. Open and filled symbols are data with and without averaging over the location of the meson source, respectively.

\section{Form factors at simulation points}

We calculate the scalar form factor $f_{0}\left(q^{2}\right)=f_{+}\left(q^{2}\right)+f_{-}\left(q^{2}\right) q^{2} /\left(M_{K}^{2}-M_{\pi}^{2}\right)$ at $q_{\max }^{2}=\left(M_{K}-\right.$ $\left.M_{\pi}\right)^{2}$ from the following double ratio [5]

$$
R\left(\Delta t, \Delta t^{\prime}\right)=\frac{C_{4}^{K \pi}\left(\Delta t, \Delta t^{\prime} ; \mathbf{0}, \mathbf{0}\right) C_{4}^{\pi K}\left(\Delta t, \Delta t^{\prime} ; \mathbf{0}, \mathbf{0}\right)}{C_{4}^{K K}\left(\Delta t, \Delta t^{\prime} ; \mathbf{0}, \mathbf{0}\right) C_{4}^{\pi \pi}\left(\Delta t, \Delta t^{\prime} ; \mathbf{0}, \mathbf{0}\right)} \underset{\Delta t, \Delta t^{\prime} \rightarrow \infty}{\longrightarrow} \frac{\left(M_{K}+M_{\pi}\right)^{2}}{4 M_{K} M_{\pi}} f_{0}\left(q_{\max }^{2}\right)^{2} .
$$

The form factors $f_{\{+, 0\}}\left(q^{2}\right)$ at $q<q_{\max }^{2}$ are calculated from [ [1, 回]

$$
\begin{aligned}
\tilde{R} & =\frac{C_{4}^{K \pi}\left(\Delta t, \Delta t^{\prime} ; \mathbf{p}, \mathbf{p}^{\prime}\right) C^{K}(\Delta t, \mathbf{0}) C^{\pi}\left(\Delta t^{\prime}, \mathbf{0}\right)}{C_{4}^{K \pi}\left(\Delta t, \Delta t^{\prime} ; \mathbf{0}, \mathbf{0}\right) C^{K}(\Delta t, \mathbf{p}) C^{\pi}\left(\Delta t^{\prime}, \mathbf{p}^{\prime}\right)} \rightarrow\left\{\frac{E_{K}+E_{\pi}^{\prime}}{M_{K}+M_{\pi}}+\frac{E_{K}-E_{\pi}^{\prime}}{M_{K}+M_{\pi}} \xi\left(q^{2}\right)\right\} \frac{f_{+}\left(q^{2}\right)}{f_{0}\left(q_{\max }^{2}\right)}, \\
R_{k} & =\frac{C_{k}^{K \pi}\left(\Delta t, \Delta t^{\prime} ; \mathbf{p}, \mathbf{p}^{\prime}\right) C_{4}^{K K}\left(\Delta t, \Delta t^{\prime} ; \mathbf{p}, \mathbf{p}^{\prime}\right)}{C_{4}^{K \pi}\left(\Delta t, \Delta t^{\prime} ; \mathbf{p}, \mathbf{p}^{\prime}\right) C_{k}^{K K}\left(\Delta t, \Delta t^{\prime} ; \mathbf{p}, \mathbf{p}^{\prime}\right)} \rightarrow \frac{2 p_{k}}{\left(p+p^{\prime}\right)_{k}} \frac{E_{K}+E_{K}^{\prime}}{\left(E_{K}-E_{\pi}^{\prime}\right) \xi\left(q^{2}\right)-E_{K}-E_{\pi}^{\prime}},
\end{aligned}
$$

where $E_{P}^{(\prime)}(P=\pi$ or $K)$ represents the energy of the meson $P$ with the momentum $\mathbf{p}^{(\prime)}$. Note that we can convert $f_{+}\left(q^{2}\right)$ to $f_{0}\left(q^{2}\right)$ (and vice versa) using the the ratio $\xi\left(q^{2}\right)=f_{-}\left(q^{2}\right) / f_{+}\left(q^{2}\right)$, except at $q_{\max }^{2}$ where $\tilde{R}$ and $R_{k}$ have no sensitivity to $\xi\left(q^{2}\right)$.

The use of the all-to-all propagator greatly helps us identify the plateaux of these ratios. In contrast to previous studies with $\Delta t+\Delta t^{\prime}$ kept fixed, we can take arbitrary combinations of $\Delta t$ and $\Delta t^{\prime}$. As shown in Fig. 㘞, the effective values of $f_{0}\left(q_{\max }^{2}\right)$ with different values of $\Delta t+\Delta t^{\prime}$ exhibit good consistency, which gives us confidence about our determination with sub-percent accuracy.

Another important advantage with the all-to-all propagator is that we can remarkably improve the statistical accuracy of meson correlators (and hence their ratios) by averaging over the location of the meson source $(\mathbf{x}, t)$ in Eqs. (‥2) and ([L.3)). As shown in the right two panels of Fig. W, the statistical fluctuation of $C_{\mu}^{K \pi}(\mu=4,1)$ is reduced by about a factor of 3 . This averaging enables us to achieve $\lesssim 1 \%$ accuracy for $f_{\{+, 0\}}\left(q^{2}\right)$, and $10-30 \%$ for $\xi\left(q^{2}\right)$. 

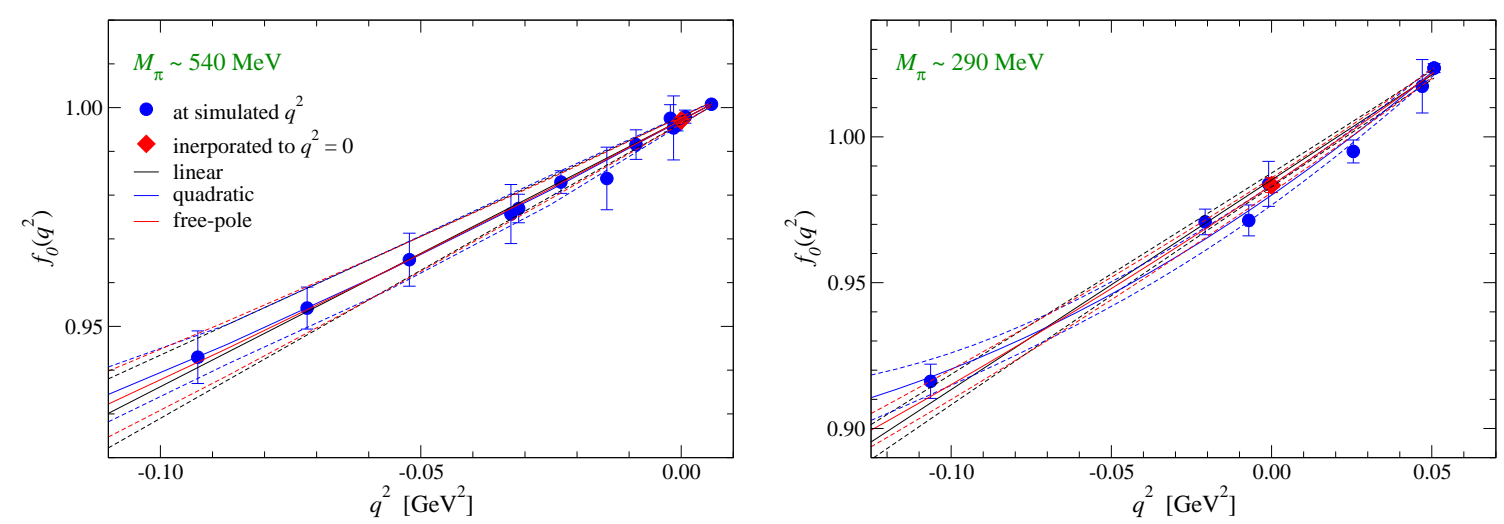

Figure 2: Scalar form factor $f_{0}\left(q^{2}\right)$ as a function of $q^{2}$. Left and right panels show data at our heaviest and lightest pion masses, respectively. We also plot interpolations to $q^{2}=0$ with various parametrization forms together with $f_{0}(0)$ obtained using the pole ansatz (diamond).

\section{3. $q^{2}$ dependence}

We plot our results for $f_{0}\left(q^{2}\right)$ as a function of $q^{2}$ in Fig. [. In this study, we simulate small values of $\left|q^{2}\right|$ by using TBCs to precisely determine $f_{+}(0)\left(=f_{0}(0)\right)$. The data show small curvature in our region of $q^{2}$ and are well described by any of the following parametrization forms

$$
f_{0}\left(q^{2}\right)=f_{0}(0)\left(1+c_{0} q^{2}\right), \quad f_{0}\left(q^{2}\right)=f_{0}(0)\left(1+c_{0} q^{2}+c_{1} q^{4}\right), \quad f_{0}\left(q^{2}\right)=\frac{f_{0}(0)}{1-q^{2} / M_{\text {pole }}^{2}}
$$

which have also been used in the analyses of experimental data. In this preliminary report, we determine the normalization $f_{0}(0)$ and its slope $f_{0}^{\prime}(0)$ using the pole form. The uncertainty due to this parametrization is estimated by the largest deviation among the results of the above three interpolations. We note that this uncertainty is similar to or smaller than the statistical error.

The situation is similar for $f_{+}\left(q^{2}\right)$, which is plotted in the left panel of Fig. B]. We observe that $f_{+}(0)$ is in good agreement with $f_{0}(0)$ as expected, while the latter has slightly smaller uncertainty due to a better control of its interpolation to $q^{2}=0$ with the accurate data of $f_{0}\left(q_{\max }^{2}\right)$. We therefore use $f_{0}(0)$ as the normalization of the vector form factor in the following analysis.
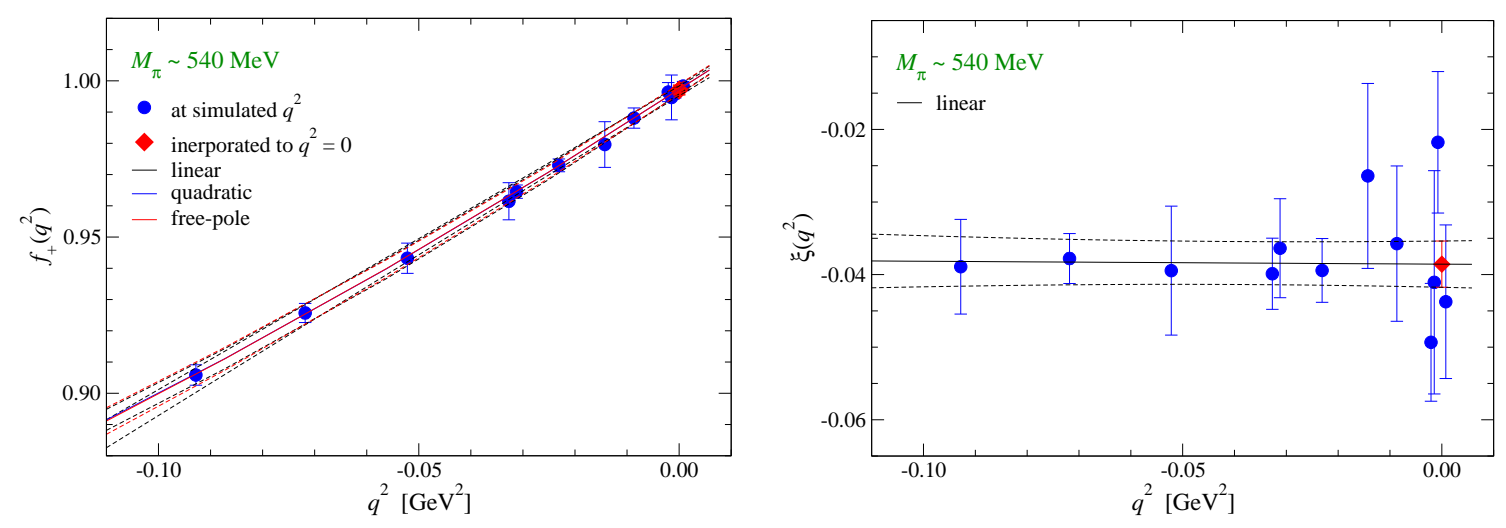

Figure 3: Interpolation of $f_{+}\left(q^{2}\right)$ (left panel) and $\xi\left(q^{2}\right)$ (right panel) as a function of $q^{2}$. 

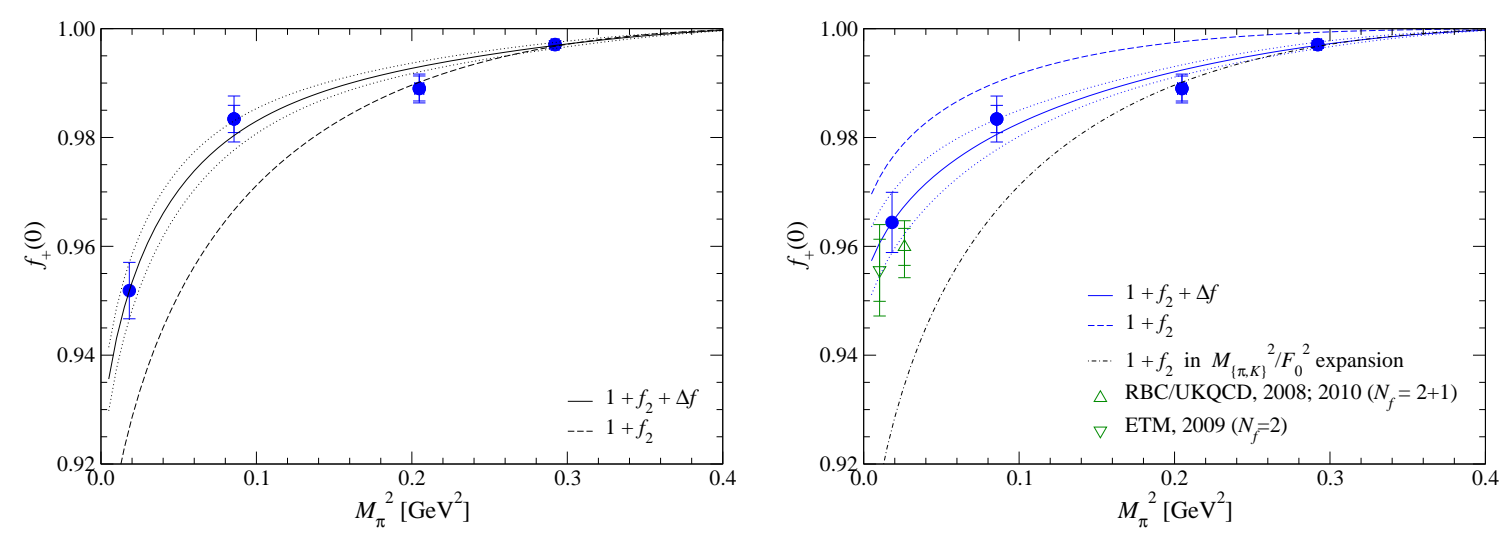

Figure 4: Chiral extrapolations of $f_{+}(0)$. The left and right panels show extrapolations based on the chiral expansion in terms of $M_{\{\pi, K\}}^{2} / F_{0}^{2}$ and $M_{\{\pi, K\}}^{2} / F_{\pi}^{2}$, respectively. In the right panel, we also plot $f_{+}(0)$ from recent calculations in $N_{f}=2+1$ [8] and $N_{f}=2$ [Q] QCD.

We plot $\xi\left(q^{2}\right)$ as a function of $q^{2}$ in the right panel of Fig. [1]. Our data show a very mild dependence on $q^{2}$ with our statistical accuracy of $\lesssim 30 \%$. Note also that the leading order (LO) analytic terms in the chiral expansion of $\xi\left(q^{2}\right)$ are independent of $q^{2}$, since $\xi\left(q^{2}\right)$ vanishes as $\propto m_{s}-m_{u d}$ in the $S U(3)$ symmetric limit [ [ $]$. We interpolate $\xi\left(q^{2}\right)$ to $q^{2}=0$ using a linear fit.

\section{Chiral extrapolation}

Figure $\mathbb{t}$ shows our chiral extrapolations of $f_{+}(0)$ based on $S U(3)$ ChPT. In this preliminary report, we employ the fitting form

$f_{+}(0)=1+f_{2}+\Delta f, \quad f_{2}=\frac{3}{2}\left(H_{K \pi}+H_{K \eta}\right)$,

$H_{P Q}=-\frac{M_{P}^{2}+M_{Q}^{2}}{128 \pi^{2} F_{0}^{2}}\left(1+\frac{2 M_{P}^{2} M_{Q}^{2}}{M_{P}^{4}-M_{Q}^{4}} \ln \left[\frac{M_{Q}^{2}}{M_{P}^{2}}\right]\right), \quad \Delta f=\left(\frac{M_{K}^{2}-M_{\pi}^{2}}{F_{0}^{2}}\right)^{2}\left\{c_{0}+c_{1} \frac{M_{K}^{2}+M_{\pi}^{2}}{F_{0}^{2}}\right\}$

where $f_{2}$ represents the next-to-leading order (NLO) contribution [ $\square$ ], and the LO relation $M_{\eta}^{2}=$ $\left(4 M_{K}^{2}-M_{\pi}^{2}\right) / 3$ is used to evaluate $H_{K \eta}$. We also include the higher order analytic correction $\Delta f$ with $c_{\{0,1\}}$ treated as fit parameters. The Ademollo-Gatto theorem [एँ] guarantees that $f_{2}$ consists only of the chiral logarithms with the single low-energy constant (LEC) $F_{0}$, that is the decay constant in the chiral limit of three flavors. Note that Eqs. (4. (ل) and (4.2) can be considered as an expansion in terms of $M_{\{\pi, K\}}^{2} / F_{0}^{2}$.

We obtain the extrapolation in the left panel of Fig. 团 by using $F_{0}=52.5(5.1)_{\text {stat }}$ determined from our study of the meson decay constants [W]. The convergence of the chiral expansion at the physical quark mass is $f_{+}(0)=1-0.073\left[f_{2}\right]+0.025(6)[\Delta f]$ in contrast to the conventional wisdom that $f_{2}$ is only few percent correction and $\Delta f$ is even smaller. This is because our estimate of $F_{0}$ is significantly smaller than the phenomenological value $F_{0}=87.7 \mathrm{MeV}$ [ए2] and enhances the chiral corrections $\propto M_{\{\pi, K\}}^{2 n} / F_{0}^{2 n}$. Note, however, that the phenomenological estimate involves large $N_{c}$ assumptions, which are not consistent with recent experimental data of the $K_{l 4}$ decays [[13]]. 

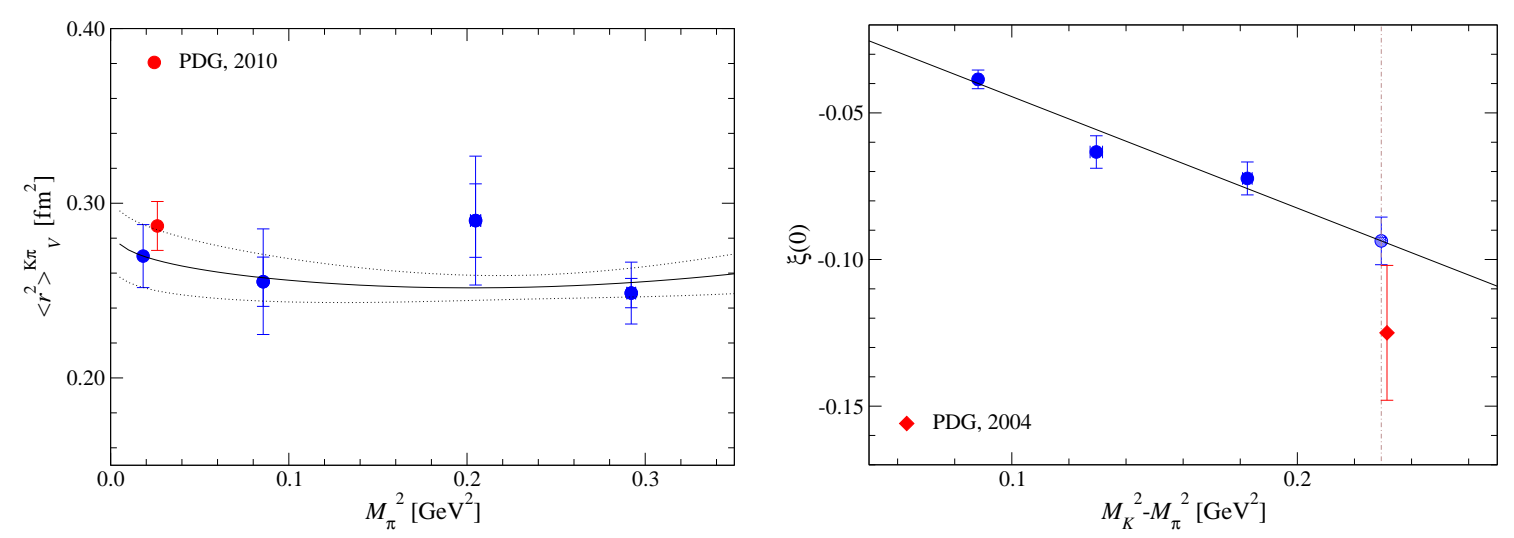

Figure 5: Chiral extrapolation of $\left\langle r^{2}\right\rangle_{V}^{K \pi}$ (left panel) and $\xi(0)$ (right panel).

The convergence can be improved by switching the expansion parameter to $M_{\{\pi, K\}}^{2} / F_{\pi}^{2}$ as we already demonstrated in our study of $M_{\pi}$ and $F_{\pi}$ in $N_{f}=2$ QCD [14]]..$^{1}$ The right panel of Fig. (1t shows the extrapolation using Eqs. (4. (ل) - (4.2) rewritten in terms of $M_{\{\pi, K\}}^{2} / F_{\pi}^{2}$. We obtain a more convergent expansion $f_{+}(0)=0.964(6)=1-0.023-0.013(6)$ without a subtle cancellation between $f_{2}$ and $\Delta f$. Note also that recent calculations in $N_{f}=2+1$ [ [8] and $N_{f}=2$ [Q] QCD are consistent with this extrapolation.

Our chiral extrapolation of the normalized slope $\left\langle r^{2}\right\rangle_{V}^{K \pi}=6 f_{+}^{\prime}(0) / f_{+}(0)$ is shown in the left panel of Fig. [5. We employ the NLO expression [[]]

$$
\begin{aligned}
\left\langle r^{2}\right\rangle_{V}^{K \pi} & =\frac{12 L_{9}^{r}}{F_{\pi}^{2}}-\frac{3}{64 \pi^{2} F_{\pi}^{2}}\left\{h\left(\frac{M_{\pi}^{2}}{M_{K}^{2}}\right)+h\left(\frac{M_{\eta}^{2}}{M_{K}^{2}}\right)+\frac{2}{3} \ln \left[\frac{M_{\pi}^{2}}{\mu^{2}}\right]+\frac{5}{3} \ln \left[\frac{M_{K}^{2}}{\mu^{2}}\right]+\ln \left[\frac{M_{\eta}^{2}}{\mu^{2}}\right]\right\}, \\
h(x) & =\frac{x^{3}-3 x^{2}-3 x+1}{2(x-1)^{3}} \ln [x]+\frac{1}{2}\left(\frac{x+1}{x-1}\right)^{2}-\frac{1}{3}, \quad \mu=M_{\rho},
\end{aligned}
$$

plus a higher order analytic correction. Note that $\left\langle r^{2}\right\rangle_{V}^{K \pi}$ has the NLO analytic term with a LEC $L_{9}^{r}$ in contrast to $f_{+}(0)$. As shown in Fig. [1, our data are well described by this form and the extrapolated value is in good agreement with the experiment [ए]]. Our estimate $L_{9}^{r} \times 10^{3}=4.1$ (3) is slightly smaller than a phenomenological estimate 5.9(4) [ㅁ]], though the error of our preliminary result is statistical only.

The right panel of Fig. [ shows our results for $\xi(0)$ as a function of the $S U(3)$ breaking parameter $M_{K}^{2}-M_{\pi}^{2}$. In this preliminary analysis, we parametrize the quark mass dependence of $\xi(0)$ by a simple linear form

$$
\xi(0)=d_{0}+d_{1}\left(M_{K}^{2}-M_{\pi}^{2}\right),
$$

which is motivated from the ChPT expression of the leading analytic terms $\propto M_{K}^{2}-M_{\pi}^{2}$ [四. Our data are well fitted to this form as shown in Fig. [. We obtain $d_{0}=-0.006(8)$ confirming that $\xi(0)$ vanishes in the $S U(3)$ symmetric limit, as expected. The extrapolation to the physical point yields $\xi(0)=-0.094(8)$ which is consistent with the experimental value $-0.125(23)$ [ए8].

\footnotetext{
${ }^{1}$ See also Ref. [I5]] for a resummation of effects of sea strange quarks, which could be a source of the large deviation between $F_{\pi}$ and $F_{0}$.
} 


\section{Summary}

In this article, we report on our calculation of the kaon semileptonic form factors. Their normalizations and slopes at $q^{2}=0$ are precisely calculated by using the all-to-all propagator and TBCs. We observe a good consistency of $\left\langle r^{2}\right\rangle_{V}^{K \pi}$ and $\xi(0)$ with experimental results.

The choice of the expansion parameter is an important issue on the convergence of the chiral expansion of $f_{+}(0)$. We note that the small value of $F_{0}$ also enhances the chiral correction to other observables, such as the pion and kaon charge radii [[]]. The large deviation $F_{\pi}-F_{0}$ can mainly come from effects of sea strange quarks, which are difficult to estimate solely from experimental data. A more precise determination of $F_{0}$ is an important task of lattice QCD to assess the reliability of the chiral expansion based on $S U(3)$ ChPT.

Our calculations at different values of $m_{u d}$ and $m_{s}$ are underway to study systematics of the chiral extrapolations. It is also interesting to apply two-loop ChPT formulae to our data. Although the formulae are complicated, exact chiral symmetry forbids additional terms due to finite lattice spacings and provides us a theoretically clean comparison at the higher order.

Numerical simulations are performed on Hitachi SR11000 and IBM System Blue Gene Solution at High Energy Accelerator Research Organization (KEK) under a support of its Large Scale Simulation Program (No. 09/10-09). This work is supported in part by the Grants-in-Aid for Scientific Research (No. 21674002, 21684013, 23105710), the Grant-in-Aid for Scientific Research on Innovative Areas (No. 2004: 20105001, 20105002, 20105003, 20105005), and the HPCI Strategic Program of the Ministry of Education, Culture, Sports, Science and Technology.

\section{References}

[1] S.Aoki et al. (JLQCD and TWQCD Collaborations), Phys. Rev. D 80, 034508 (2009).

[2] T. Kaneko et al. (JLQCD Collaboration), PoS Lattice 2010, 146 (2010).

[3] G.S. Bali et al. (SESAM Collaboration), Phys. Rev. D 71, 114513 (2005); J. Foley et al. (TrinLat Collaboration), Comput. Phys. Commun 172, 145 (2005).

[4] P.F. Bedaque, Phys. Lett. B 593, 82 (2004).

[5] D. Bećirević et al., Nucl. Phys. B 705, 339 (2005).

[6] N. Tsutsui et al. (JLQCD Collaboration), PoS LAT2005, 357 (2005); C. Dawson et al. (RBC Collaboration) Phys. Rev. D 74, 114502 (2006).

[7] J. Gasser and H. Leutwyler, Nucl. Phys. B 250, 517 (1985).

[8] P.A. Boyle et al. (RBC and UKQCD Collaborations), Phys. Rev. Lett. 100, 141601 (2008).

[9] V. Lubicz et al. (ETM Collaboration), Phys. Rev. D 80, 111502 (2009).

[10] M. Ademollo and R. Gatto, Phys. Rev. Lett. 13, 264 (1964).

[11] J. Noaki et al. (JLQCD and TWQCD Collaborations), PoS Lattice 2010, 117 (2010).

[12] G. Amoros, J. Bijnens and P. Talavera, Nucl. Phys. B 602, 87 (2001).

[13] J. Bijnens and I. Jemos, arXiv:1103.5945 [hep-ph].

[14] J. Noaki et al. (JLQCD and TWQCD Collaborations), Phys. Rev. Lett. 101, 202004 (2008).

[15] S. Descotes-Genon et al., Eur. Phys. J. C 52, 141 (2007).

[16] K. Nakamura et al. (Particle Data Group), J. Phys. G 37, 075021 (2010).

[17] J. Bijnens and P. Talavera, JHEP 03, 046, (2002).

[18] S. Eidelman et al. (Particle Data Group), Phys. Lett. B 592, 1 (2004). 